\title{
Representación gráfica del riesgo de mortalidad neonatal en un centro perinatal regional en Mérida, Yucatán, México
}

\author{
Lorenzo 0 sorno-Covarrubias, Ped Neonat, ${ }_{1}^{(1)}$ Gabriela Vela-Urtecho, M Ped, ${ }^{(2)}$
}

Jorge Dávila-Velázquez, M en C. . $^{(3)}$

Osorno-Covarrubias L, Vela-Urtecho G, Dávila-Velázquez J.
Representación gráfica del riesgo
de mortalidad neonatal en un centro perinatal
regional en Mérida,Yucatán, México.
Salud Publica Mex 2002;44:345-348.

El texto completo en inglés de este artículo está disponible en: http://www.insp.mx/salud/index.html

\section{Resumen}

Objetivo. Determinar el riesgo de mortalidad neonatal por edad gestacional y el peso al nacer. Material y métodos. Se estudió una cohorte de 19668 neonatos que egresaron entre el 1 de enero de 1995 y el 31 de octubre de 1999 del Centro Médico Nacional Ignacio García Téllez, del tercer nivel de atención perinatal del Instituto Mexicano del Seguro So cial de la Península deYucatán. Se registraron el peso al nacer, edad gestacional y condición de egreso. Se calculó el riesgo absoluto (RA) de mortalidad para cada semana de edad gestacional y grupo de peso. Resultados El RA de mortalidad observado en neonatos de entre 34 a 44 semanas y peso mayor o igual a $2250 \mathrm{~g}$ fue de $0.4 \%$, de $15 \%$ para aquellos de entre 26 a 32 semanas con peso mayor o igual a $1000 \mathrm{~g}$, y de $73 \%$ para los de entre las 26 a las 34 semanas, con peso al nacimiento de entre 750 y $1000 \mathrm{~g}$. Conclusiones. El RA de mortalidad neonatal aumentó a menor edad gestacional y peso. Los datos pueden ser utilizados como valores de referencia para nuestro hospital y para comparación con otros hospitales. El texto completo en inglés de este artículo está disponible en:http://www.insp.mx/ salud/index.html

Palabras clave: recién nacido; riesgo de mortalidad neonatal; edad gestacional; peso al nacer; M éxico
Osorno-Covarrubias L,Vela-Urtecho G, Dávila-Velázquez J. The graphical display of neonatal mortality risk at a regional perinatal center in Merida, Yucatan, Mexico. The joint effect of birth weight and gestational age. Salud Publica Mex 2002;44:345-348.

The English version of this paper

is available at: http://www.insp.mx/salud/index.html

\section{A bstract}

Objective. To determine the neonatal mortality risk according to gestational age and birth weight. Material and Methods The cohort consisted of 19668 newborns of Centro Médico Nacional (N ational Medical Center) Ignacio García Téllez, a tertiary level healthcare institution of the Instituto Mexicano del Seguro Social (Mexican Institute of Social Security, IMSS) of the Yucatan Peninsula. All newbo rns discharged from the hospital between January $1^{\text {st }}, 1995$ and 0 ctober $31^{\text {st }}, 1999$ were included in the study. Birth weight, gestational age, and conditions upon discharge were recorded. Absolute risk (AR) of mortality was calculated for each week-of-gestation- and birth group. Results $0 \mathrm{~b}$ served AR in newborns 34 to 44 weeks of gestational age and weighing at least $2250 \mathrm{~g}$ was 0.4 , while that for those 26 to 32 weeks of gestational age and weighing between $1000 \mathrm{~g}$ was $15 \%$. Conclusions AR of neonatal mortality increased inversely proportional to gestational age and birth weight. These data can be used as reference values for our hospital and for comparison with other hospitals. The English version of this paper is available at: http://www.insp.mx/ salud/index.html

Key words: infant-newborn; neo natal mortality risk; gestational-age; birth weight; M exico

(1) Departamento Clínico de Neonatología del Centro Médico Nacional "Ignacio García Téllez" del Instituto Mexicano del Seguro Social, Mérida, Yucatán, México.

(2) Centro Médico Nacional "Ignacio GarcíaTéllez", Mérida, Yucatán, México.

(3) Unidad de Investigación en Epidemiología C línica, Centro Médico N acional "Ignacio García Téllez" Mérida,Yucatán, México.

Fecha de recibido: 1 de febrero de 2001 • Fecha de aprobado: 7 de junio de 2002

Solicitud de sobretiros: Dr. Lorenzo 0 sorno-C ovarrubias. Servicio de N eonatología del Centro Médico N acional "Ignacio GarcíaTéllez". Calle 34 Núm. 439 x 41, ex terrenos del Fénix, Colonia Industrial 97150, Mérida,Yucatán, México.

Correo electrónico:I_osorno@ pibil.finred.com.mx 
L as tasas de mortalidad neonatal permiten valorar la calidad de la atención perinatal prestada en los servicios de los centros hospitalarios. La mortalidad neonatal varía de acuerdo con la edad gestacional y peso al nacimiento; en 1955 Schlesinger observó una mayor mortalidad neonatal a menor peso y edad gestacional, y enfatizó la importancia de tomar en cuenta ambos parámetros, y no sólo la prematurez o el bajo peso. ${ }^{1}$

En 1972 Battaglia y colaboradores publicaron valores de riesgo de mortalidad neonatal de acuerdo con el peso para la edad gestacional en neonatos de 24 a 42 semanas, y de 500 a 4500 gramos de peso; los valores obtenidos en ese estudio se muestran sobrepuestos en las curvas de crecimiento intrauterino y con celdas que representan cada semana de edad gestacional y grupo de peso de 250 gramos. $^{2}$ En este estudio se observó que el menor riesgo de mortalidad se encuentra en los neonatos de 40 semanas y con peso de 2500 a 3800 gramos (riesgo de 0.1 a $0.9 \%$ ), pero este riesgo se incrementa de seis a ocho veces más si el peso al nacer es mayor de 4000 gramos y nueve veces si el peso es menor de 1750 gramos. En 1982 Koops y Battaglia actualizaron las tablas de riesgo de mortalidad neonatal presentándola en forma de bloques agrupados según las tendencias de riesgo de los grupos de peso y edad gestacional. $^{3}$

Existen múltiples estudios que describen el riesgo de mortalidad neonatal en diversas circunstancias y poblaciones:4-8 ninguno de ellos incluye un rango semejante a los dos parámetros antes mencionados por Lubchenco, Battaglia y colaboradores.

El propósito de este estudio es determinar el riesgo mortalidad neonatal de acuerdo con la combinación de peso al nacimiento y edad gestacional.

\section{Material y métodos}

Se estudió una cohorte de neonatos nacidos en el Centro Médico Nacional Ignacio García Téllez (CMN) del Instituto Mexicano del Seguro Social (IMSS), de la Ciudad de Mérida, Yucatán, México, los cuales fueron seguidos hasta su egreso, durante el periodo comprendido entre el 1 de enero de 1995 al 31 de octubre de 1999. No hubo seguimiento de los neonatos egresados antes de los 28 días. No se incluyeron mortinatos, neonatos fallecidos después de los 28 días de vida, ni aquellos que fueron transportados de otras unidades. Se eliminaron los neonatos cuyos datos fueron incompletos, y a quienes fueron transportados a otras unidades.
El CMN Ignacio García Téllez del IMSS funciona como centro de atención perinatal de tercer nivel para toda la Península de Yucatán, y atiende los embarazos de alto y bajo riesgo procedentes de las clínicas de medicina familiar de la zona norte y oriente de la ciudad de Mérida.

Se capturó la información en dBase IV. Se registró fecha de última menstruación (FUM), fecha de nacimiento del neonato, edad gestacional por la FUM por los métodos de Capurro ${ }^{9}$ o Ballard, ${ }^{10}$ peso al nacimiento, edad al egreso, condición de egreso y fecha de egreso. La validez de la edad gestacional se obtuvo con la correlación entre la FUM y Ballard; en caso de que la edad gestacional, calculada por la FUM, fuera mayor o menor a dos semanas que la calculada por los métodos de Capurro o Ballard se tomó esta última como referencia.

Se hizo el cálculo de riesgo absoluto (RA) de mortalidad por cada semana de edad gestacional y grupos de peso de 250 g; para simplificación en la presentación de los datos se redondearon las cifras decimales a la unidad más próxima cuando el valor fue mayor de 2. Estos datos fueron procesados en el paquete estadístico del programa SPSS 8.0 para Windows.

\section{Resultados}

En el lapso de 1995 a 1999 se registraron 26380 recién nacidos vivos, con una pérdida de 901 casos (4.4\%). La base de datos de 1997 se excluyó del análisis por pérdida de $42 \%$ de los casos. Por lo tanto se incluyeron 20091 recién nacidos vivos.

Del total de neonatos captados en la base de datos se eliminaron $36(0.1 \%)$ por datos incompletos o inconsistentes en el peso al nacimiento, 350 casos (1.7\%) por la edad gestacional y $37(0.18 \%)$ nacidos en su hogar, quedando para el análisis 19668 casos.

El número de neonatos de más de 500 g y 24 semanas o más de vida que fallecieron en los primeros 28 días de nacidos fue de 209/19 668 (10.6/1 000 nacidos vivos).

El RA de mortalidad para los neonatos de acuerdo con su peso y edad gestacional está representado en el cuadro I, cada rectángulo corresponde a una semana y a rangos de peso de $250 \mathrm{~g}$. El número de muertes neonatales, el total de recién nacidos vivos para cada grupo de peso y edad gestacional se indica en el cuadro II.

El RA de mortalidad observado para los neonatos de entre 34 y 44 semanas con peso al nacer mayor o igual a 2250 gramos fue de $0.4 \%$, de $15 \%$ para aque- 


\section{Cuadro I}

Riesgo porcentual de mortalidad neonatal. Mérida,Yucatán, México, 1995-1999

\begin{tabular}{|c|c|c|c|c|c|c|c|c|c|c|c|c|c|c|c|c|c|c|c|c|c|}
\hline 4500 & & & & & & & & & & & & & & 0 & 0 & 0 & 0 & 0 & 0 & & \\
\hline 4250 & & & & & & & & & & & & & 0 & 0 & 0 & 0 & 0 & 0 & 0 & 0 & \\
\hline 4000 & & & & & & & & & & & & 0 & 0 & 0 & 0 & 0 & 1.2 & 0 & 0 & & 0 \\
\hline 3750 & & & & & & & & & 0 & & 0 & 0 & 0 & 0 & 0 & 0 & 0.3 & 0 & 0 & 0 & 0 \\
\hline 3500 & & & & & & & & & & & 0 & 17 & 0 & 0 & 0.2 & 0 & 0.1 & 0 & 0 & 0 & \\
\hline 3250 & & & & & & & 0 & & 0 & 0 & 0 & 0 & 2 & 0 & 0 & 0.3 & 0.3 & 0 & 0 & 0 & 0 \\
\hline 3000 & & & & & & & & & 0 & 0 & 0 & 0 & 0 & 0.3 & 0.2 & 0.1 & 0.4 & 0 & 0 & 0 & 0 \\
\hline 2750 & & & & & & & 0 & 0 & 0 & 33 & 6 & 0 & 0.7 & 0.6 & 0 & 0.1 & 0.1 & 0 & 0 & 0 & 0 \\
\hline 2500 & & & & & & & 0 & 0 & 0 & 11 & 0 & 0 & 0.6 & 0.4 & 0.4 & 0.5 & 0 & 0 & 2 & 0 & 0 \\
\hline 2250 & & & & & & 0 & 0 & 0 & 0 & 14 & 0 & 0 & 3 & 0.7 & 0 & 1.5 & 4 & 0 & 0 & 0 & \\
\hline 2000 & & & & 100 & 0 & & 0 & 0 & 8 & 4 & 0 & 2 & 2 & 2 & 1.4 & 4 & 0 & 0 & 0 & & \\
\hline 1750 & & & & 100 & & 0 & 0 & 36 & 5 & 5 & 4 & 6 & 3 & 0 & 5 & 9 & 14 & 0 & 0 & 0 & \\
\hline 1500 & & & & & 0 & 20 & 22 & 34 & 9 & 6 & 0 & 8 & 0 & 11 & 29 & 50 & 0 & & 0 & & \\
\hline 1250 & & & & 0 & 11 & 62 & 20 & 11 & 15 & 10 & 6 & 11 & 14 & & 20 & 50 & 0 & & 0 & & \\
\hline 1000 & 100 & 100 & 0 & 75 & 45 & 37 & 26 & 6 & 25 & 0 & 50 & 0 & 0 & 100 & & 33 & & 0 & & & \\
\hline 750 & 67 & & 67 & 82 & 56 & 80 & 75 & 50 & & 100 & & & 0 & & & & & & & & \\
\hline \multirow[t]{2}{*}{500} & 100 & 86 & 80 & 100 & 80 & 100 & 100 & & & & 100 & & & & & & & & & & \\
\hline & 24 & 25 & 26 & 27 & 28 & 29 & 30 & 31 & 32 & 33 & 34 & 35 & 36 & 37 & 38 & 39 & 40 & 41 & 42 & 43 & 44 \\
\hline
\end{tabular}

Porcentaje de riesgo de mortalidad neonatal de acuerdo con el peso al nacimiento y la edad gestacional en 19698 recién nacidos en el Centro Médico N acional Ignacio García Téllez, de Mérida,Yucatán, de 1995 a 1999

\section{Cuadro II}

Número de defunciones/total de pacientes. Mérida, Yucatán, México, 1995-1999

\begin{tabular}{|c|c|c|c|c|c|c|c|c|c|c|c|c|c|c|c|c|c|c|c|c|c|}
\hline 4500 & & & & & & & & & & & & & & $0 / 4$ & $0 / 7$ & $0 / 9$ & $0 / 20$ & $0 / 5$ & $0 / 6$ & $0 / 1$ & \\
\hline 4250 & & & & & & & & & & & & & $0 / 1$ & $0 / 4$ & $0 / 22$ & $0 / 33$ & $0 / 46$ & $0 / 23$ & $0 / 4$ & $0 / 1$ & \\
\hline 4000 & & & & & & & & & & & & $0 / 1$ & $0 / 2$ & $0 / 11$ & $0 / 57$ & $0 / 126$ & $2 / 163$ & $0 / 62$ & $0 / 16$ & & $0 / 1$ \\
\hline 3750 & & & & & & & & & $0 / 1$ & & $0 / 2$ & $0 / 4$ & $0 / 6$ & $0 / 27$ & $0 / 126$ & $0 / 291$ & $1 / 374$ & $0 / 155$ & $0 / 45$ & $0 / 4$ & $0 / 1$ \\
\hline 3500 & & & & & & & & & & & $0 / 1$ & $1 / 6$ & $0 / 19$ & $0 / 71$ & $1 / 431$ & $0 / 697$ & $1 / 866$ & $0 / 300$ & $0 / 84$ & $0 / 3$ & \\
\hline 3250 & & & & & & & $0 / 1$ & & $0 / 1$ & $0 / 2$ & $0 / 12$ & $0 / 11$ & $1 / 45$ & $0 / 177$ & $0 / 688$ & $3 / 1011$ & $3 / 1151$ & $0 / 352$ & $0 / 115$ & $0 / 10$ & $0 / 4$ \\
\hline 3000 & & & & & & & & & $0 / 5$ & $0 / 4$ & $0 / 15$ & $0 / 25$ & $0 / 79$ & $1 / 324$ & $2 / 1043$ & $2 / 1345$ & $6 / 1365$ & $0 / 409$ & 0 & $0 / 18$ & $0 / 6$ \\
\hline 2750 & & & & & & & $0 / 1$ & $0 / 2$ & $0 / 3$ & $2 / 6$ & $1 / 18$ & $0 / 42$ & $1 / 148$ & $2 / 338$ & $0 / 849$ & $1 / 811$ & $1 / 732$ & $0 / 288$ & $0 / 81$ & $0 / 8$ & $0 / 3$ \\
\hline 2500 & & & & & & & $0 / 1$ & $0 / 1$ & $0 / 4$ & $1 / 9$ & $0 / 30$ & $0 / 77$ & $1 / 172$ & $1 / 276$ & $2 / 468$ & $2 / 397$ & $0 / 344$ & $0 / 99$ & $1 / 46$ & $0 / 2$ & $0 / 3$ \\
\hline 2250 & & & & & & $0 / 1$ & $0 / 1$ & $0 / 5$ & $0 / 5$ & $3 / 22$ & $0 / 44$ & $0 / 66$ & $4 / 136$ & $1 / 148$ & $0 / 162$ & $2 / 131$ & $3 / 81$ & $0 / 35$ & $0 / 15$ & $0 / 2$ & \\
\hline 2000 & & & & $1 / 1$ & $0 / 2$ & & $0 / 2$ & $0 / 4$ & $2 / 24$ & $1 / 24$ & $0 / 45$ & $1 / 43$ & $2 / 93$ & $1 / 48$ & $1 / 72$ & $2 / 54$ & $0 / 29$ & $0 / 3$ & $0 / 1$ & & \\
\hline 1750 & & & & $1 / 1$ & & $0 / 2$ & $0 / 6$ & $4 / 11$ & $2 / 41$ & $2 / 40$ & $2 / 51$ & $2 / 33$ & $1 / 34$ & $0 / 28$ & $1 / 21$ & $1 / 11$ & $2 / 14$ & $0 / 2$ & $0 / 2$ & $0 / 1$ & \\
\hline 1500 & & & & & $0 / 1$ & $1 / 5$ & $2 / 9$ & $9 / 26$ & $2 / 23$ & $1 / 17$ & $0 / 22$ & $2 / 24$ & $0 / 10$ & $1 / 9$ & $2 / 7$ & $1 / 2$ & $0 / 1$ & & $0 / 3$ & & \\
\hline 1250 & & & & $0 / 4$ & $1 / 9$ & $5 / 8$ & $5 / 25$ & $2 / 18$ & $3 / 20$ & $1 / 10$ & $1 / 16$ & $1 / 9$ & $1 / 7$ & & $1 / 5$ & $1 / 2$ & $0 / 1$ & & $0 / 1$ & & \\
\hline 1000 & $1 / 1$ & $1 / 1$ & $0 / 3$ & $3 / 4$ & $5 / 11$ & $3 / 8$ & $6 / 23$ & $1 / 15$ & $2 / 8$ & $0 / 1$ & $1 / 2$ & $0 / 4$ & $0 / 1$ & $1 / 1$ & & $1 / 3$ & & $0 / 1$ & & & \\
\hline 750 & $2 / 3$ & & $4 / 6$ & 9/11 & $5 / 9$ & $4 / 5$ & $3 / 4$ & $1 / 2$ & & $3 / 3$ & & & $0 / 1$ & & & & & & & & \\
\hline \multirow[t]{2}{*}{500} & $6 / 6$ & $6 / 7$ & $4 / 5$ & $3 / 3$ & $4 / 5$ & $2 / 2$ & $2 / 2$ & & & & $1 / 1$ & & & & & & & & & & \\
\hline & 24 & 25 & 26 & 27 & 28 & 29 & 30 & 31 & 32 & 33 & 34 & 35 & 36 & 37 & 38 & 39 & 40 & 41 & 42 & 43 & 44 \\
\hline
\end{tabular}

N úmero de muertes neonatales / el total de recién nacidos para cada grupo de peso y edad gestacional en 19698 recién nacidos en el Centro Médico N acional Ignacio García Téllez, de Mérida,Yucatán, de 1995 a 1999 
llos entre las 26 y 32 semanas con peso por arriba o igual a 1000 gramos, y de $73 \%$ para aquellos entre las 26 y 34 semanas con peso inferior a 1000 gramos.

\section{Discusión}

El RA de mortalidad aumenta a menor edad gestacional y peso, como está descrito en la literatura. Comparando este estudio con los datos del Centro Médico de la Universidad de Colorado, que incluyó neonatos desde 1958 a $1969,{ }^{5}$ nuestros resultados muestran una mortalidad más baja en neonatos pretérmino (cuadro I), sin embargo, ligeramente más alta que la reportada por el mismo grupo en 1982.

La tasa de mortalidad neonatal observada en nuestra población fue de 10.6/1 000 nacidos vivos (n v), la reportada por Rodríguez y Enríquez, ${ }^{11}$ en Torreón, Coahuila, México, fue de 12.9/1 000 n v y la observada en el año de 1995 en Estados Unidos de América $^{12}$ fue de 4.9/1 $000 \mathrm{n}$ v.

Las datos proporcionados en este estudio ofrecen un cálculo del riesgo de mortalidad neonatal en toda la gama de pesos al nacer y edades gestacionales, lo cual puede ser útil para dar información a los familiares con recién nacidos en condiciones semejantes, y como comparación tanto de la propia unidad en el tiempo, como con otras unidades en escala nacional e internacional.

En los resultados existe una subestimación de la mortalidad neonatal debido a que no se realizó el seguimiento de los neonatos egresados antes de los 28 días, aunque conocemos que la mortalidad posterior al egreso de los neonatos es baja.

Concluimos que el riesgo de mortalidad neonatal es inversamente proporcional al peso y a la edad ges- tacional, sin embargo observamos mayor mortalidad comparada con la observada por el grupo de la Universidad de Colorado en 1982.

\section{Referencias}

1. Schlesinger E,Allaway N . The combined effect of birth weight and length of gestation on neonatal mortality among single premature infants. Pediatrics 1955;15:698.

2. Lubchenco LO, Searls DT, Brazie JB. N eonatal mortality rate: Relationship to birthweight and gestational age.J Pediatr 1972;81:814.

3. Koops BL, Morgan LJ, Battaglia FC. N eonatal mortality risk in relation to birth weight and gestational age: Update.J Pediatr 1982;101:969-977.

4. Jasso GL. Relevancia de la salud perinatal en el Instituto Mexicano del Seguro Social. Bol Med Hosp Infant Mex 1997;54:54-58.

5.Tejero-Torres JC. Los límites de la viabilidad: consideraciones clínicas y éticas. Rev Esp Pediatr 1997;53:119-125.

6. Jasso GL. Introducción. En:Temas de Pediatría: N eonatología. México, D.F:A sociación Mexicana de Pediatría, Editorial Interamericana, 1996: 1-12.

7. Rivera RS, Fernández CJM, Estrada FJV, Alvarez VE. Letalidad y morbilidad en neonatos con peso menor de $1000 \mathrm{gr}$. Rev Mex Pediatr 1995;62: 88-93.

8. De la Garza Q C, González SM. Muerte perinatal. Ginecol 0 bstet Mex 1995;63:186.

9. Capurro H, Konichezky S, Fonseca D, Caldeyro-Barcia R. A simplified method of fetal maturation of the newborn infant.J Pediatr 1978;93:120. 10. Ballard JL, Khoury JC, W edig K, W ang L, Eilers-W alsman BL, Lipp-R. $\mathrm{N}$ ew Ballard Score, expanded to include extremely premature infants. J Pediatr 1991;119:417-423.

11. Rodríguez y Enríquez-de Rivera FC, Velásquez-Trejo ML, Rois-Hernández J. Mortalidad perinatal en unidades de atención médica del IMSS (Instituto Mexicano del Seguro Social) Centro Médico $\mathrm{N}$ acional de Torreón. Ginecol O bstet Mex 1998 Jul;66:297-300.

12. Lemons JA, Bauer CR, O h W, Korones SB, Papile LA, Stoll BJ et al.Very low birth weight outcomes of the $\mathrm{N}$ ational Institute of $\mathrm{C}$ hild $\mathrm{H}$ ealth and Human D evelopment N eonatal Research N etwork, January 1995 through December 1996. NICHD N eonatal Research N etwork. Pediatrics 2001 Jan;107. CD: El. 